\title{
Polisemia y cognición del verbo ir en castellano ${ }^{1}$ Polysemy and cognition of the verb "ir" in Spanish
}

Gisela Silva Escudero Universidad Peruana Cayetano Heredia, Lima, Perú gisela.silva@upch.edu.pe ORCID: https://orcid.org/0000-0003-1410-0574

\section{Resumen}

Se aborda la polisemia del verbo ir desde la perspectiva cognitiva del lenguaje. El objetivo fundamental del presente trabajo es determinar los diferentes sentidos o significados de la palabra ir. Resulta revelador definir la red de relaciones semánticas de esta forma verbal que, en principio, se asocia con realidades de tipo físico. Se detecta que, a pesar de que existen significados vinculados con otros dominios de la realidad que no son de orden físico, los mecanismos cognitivos metafóricos hacen posible la conceptualización de dominios diferentes como si se trataran del mundo material. En rigor, detectamos que se conforma una red semántica a partir de un significado central del cual irradian otros de carácter periférico.

Palabras clave Lingüística cognitiva, polisemia, ir, red semántica, espacialidad

\begin{abstract}
The polysemy of the verb ir is approached from the cognitive perspective of language. The main objective of the present work is to determine the different senses or meanings of the word $i r$. It is revealing to define the network of semantic relations of this verb form which, in principle, is associated with physical realities. It is detected that, although there are meanings linked to other domains of reality that are not of a physical order, the metaphorical cognitive mechanisms make possible the conceptualization of different domains as if they were of the material world. Strictly speaking, we detect that a semantic network is formed from a central meaning from which other peripheral meanings radiate.
\end{abstract}

Keywords Cognitive linguistics, polysemy, go, semantic network, spatiality

Fecha de envio: 14/12/2020 Fecha de aceptación: 23/4/2021 


\section{Introducción}

El presente estudio analiza el fenómeno de polisemia en el verbo de movimiento ir en castellano. La forma en que este verbo se manifiesta involucra diversos ámbitos que tradicionalmente han sido descritos como significados divergentes o como sentidos o usos que presenta alguna relación; sin embargo, estos carecen de sistematicidad y, por esa razón, consideramos importante abordar el fenómeno a través de los presupuestos de la lingüística cognitiva (LC). El estudio de la polisemia en este trabajo considera la conformación de estructuras de significado que, como se verá en las secciones posteriores, se relacionan de manera orgánica. Estas, a su vez, están motivadas por la experiencia de los hablantes, quienes usan mecanismos cognitivos para organizar la realidad, significarla y expresarla a través de vínculos semánticos que develan la necesidad de organizar la realidad. Esto quiere decir que nuestra propuesta presenta una sustentación medular en los usos reconocibles del verbo ir, el cual sustancialmente expresa desplazamiento espacial, pero que puede involucrar otras aristas relativas a dominios diferentes como el de las sensaciones, las inclinaciones ideológicas, los procesos fisiológicos internos al individuo; es decir, además del desplazamiento propio y paradigmático de este verbo, en el cual la dimensión espacial involucra el establecimiento de categorías semánticas de naturaleza físico-espacial, los significados también se organizan en torno a otros dominios semánticos. En tal sentido, la determinación de los elementos extralingüísticos que definen el sistema de relaciones de significado será importante para entender los mecanismos que interactúan en la ocurrencia de tales sistemas. Iniciaremos el desarrollo del análisis polisémico del verbo ir sustentando las razones de por qué resulta necesario el desarrollo de un marco de análisis semántico de tipo cognitivo en lo concerniente a la evaluación del comportamiento significativo de este verbo; tal análisis podría extenderse hacia otros verbos u otros fenómenos léxicos en español. El estudio de casos sirve para entender el comportamiento interlingüístico en términos tipológicos, además de incluir en el estudio del significado la consideración de la concreción lingüística antes que las abstracciones descontextualizadas y desocializadas.

\section{Justificación}

Los estudios descriptivos del significado en las lenguas naturales han estado determinados por enfoques que sustentaban el análisis más en inventarios que se explicaban a través del contexto en el que aparecían los vocablos en cuestión; sin embargo, la orientación de este tipo implicaba una interpretación del significado 
como entidad individual que en el uso adquiría sentidos de diverso tipo. La desventaja de esta propuesta era que muchos de los sentidos resultaban arbitrarios, dado que se carecía de criterios formales que permitieran establecer un sistema organizado en el que se integren los diversos conceptos de naturaleza semántica. La manifestación en enunciados concretos responde entonces a mecanismos cognitivos que resulta importante reconocer y describir. La descripción formal de estos recursos que hacen posible configurar significados en español, y que se puede extender al análisis de cualquier lengua natural, lo cual permitiría establecer tipologías semánticas, además de permitir establecer predicciones sobre la naturaleza semántica del lenguaje asociado con el movimiento, es decir, con el mundo espacial y sus elementos más relevantes. Los trabajos de análisis del tipo que presentamos en este trabajo haría posible la consideración de elementos más amplios del análisis semántico y sus potenciales aplicaciones en el proceso de enseñanza del español como segunda lengua, la traducción de textos del español a otras lenguas, etc. Por ello, consideramos que es importante entender y corroborar empíricamente la forma en que el español expresa su dinamismo (como cualquier otra lengua, claro está) a través de significados espaciales que se extienden a otros dominios derivados de la vivencia misma de los hablantes. El análisis objetivo de estos será particularmente importante para comprender también la naturaleza cognitiva de la polisemia, como fenómeno cognitivo que implica un mecanismo consistente mediante el cual los hablantes organizan a través de conceptos y expresiones que reflejan la interacción entre el lenguaje y el medio externo. Por las razones expuestas consideramos que el aporte de nuestro trabajo en la descripción del fenómeno semántico en español es relevante para comprenderlo plenamente en términos no convencionales; en otras palabras, a través de reflexiones que permitan considerar la naturaleza misma del fenómeno del uso concreto de una lengua.

\section{Planteamiento del problema}

El verbo ir en castellano presenta una serie de sentidos, muchas veces registrados como conceptos independientes, por cuanto en el uso expresan dominios no espaciales. Por ejemplo, en la Base de datos de Verbos, Alternancias de Diátesis y Esquemas Sintáctico-Semánticos del Español (Adesse) ${ }^{2}$, de la Universidad de Vigo, se proponen cinco secciones que agrupan muestras diferentes. Esta clasificación impone definiciones semánticas específicas que permite establecer clasificaciones al interior de cada una. Así, las cinco acepciones que se proponen son las siguientes ${ }^{3}$ : 


\author{
IR I: desplazamiento \\ IR II: propiedad \\ IR III: relación \\ IR IV: sensación \\ IR V: fisiología
}

El sentido más reconocido es el espacial y supone el desplazamiento de un lugar a otro. En cada uno de los casos que se proponen en este repositorio, se evidencia la relación con el dominio espacial, de manera que podría encontrarse un sentido propiamente de movilización en el espacio en expresiones como Me voy de este lugar, con una dirección indeterminada; así, también es posible que el sentido verbal de desplazamiento incluya elementos abstractos como el tiempo; por ejemplo, El tiempo se nos fue volando este año. En este último caso, la espacialidad se detecta en términos no convencionales. Sin embargo, es posible que también exprese el desenvolvimiento de algo de una determinada manera; por ello, es posible distinguir casos como Vamos armados de actitud ante la adversidad o ¿cómo vamos con el inglés? Estas expresiones son propias de un dominio no espacial. Además, en cuanto a la acepción III recogida en este repositorio, se define en el sentido de armonizar o coincidir: a mi no me va tu manera de pensar, le voy al Alianza Lima este fin de semana, le voy a la vida despreocupada. Este caso es interesante porque va alejándose del sentido paradigmático de tipo dimensional espacial. En cuanto al registro IV, también se advierte que puede significar 'gustar' con lo cual se vincula con el ámbito de las sensaciones: no me van las películas de terror, bañarme con agua fría no me va, no me va porque es muy sabihondo. Finalmente, también se detecta un caso asociado con el ámbito fisiológico; este se relaciona con la expulsión de excrementos; por ejemplo, después de comer, me vine en diarrea.

Los casos anteriores son una muestra concreta de la forma en que se explicitan los sentidos del verbo de movimiento ir, que sustancialmente se interpreta como desplazamiento, pero que puede relacionarse con otras aristas de significado que pueden resultar sumamente disímiles y carentes de una relación consistente. Por ello, formulamos los siguientes problemas:

a) ¿Qué mecanismos relacionados con el significado están involucrados en la variación de sentidos del verbo de movimiento ir en castellano? 
b) ¿Es posible definir relaciones entre los diferentes significados del verbo ir que aparecen como entidades independientes?

\section{Objetivos}

Los objetivos de nuestra propuesta son los siguientes:

\subsection{Objetivo general}

Explicar los mecanismos semánticos involucrados en la variación polisémica del verbo de movimiento ir en castellano.

\subsection{Objetivos secundarios}

a) Realizar un registro de casos en los cuales se advierta la variación de sentidos o significados del verbo de movimiento ir en castellano.

b) Determinar cuáles son los mecanismos de asociación semántico-cognitiva relativos a la organización de la polisemia del verbo de movimiento ir en castellano.

\section{Hipótesis}

La hipótesis fundamental que proponemos como conjetura explicativa de la variación de significados del verbo ir en castellano es la siguiente y responde a los objetivos propuestos:

Los significados polisémicos relativos al verbo de movimiento ir en castellano se explican por la conformación sistemática de una red cuyo sentido central se vincula con el dominio espacial.

\section{Metodología}

Los procedimientos metodológicos que asumiremos para la concreción del presente estudio sobre el verbo de movimiento ir en castellano se relacionan con la recopilación de data mediante dos fuentes fundamentales: el banco de datos Adesse, en el cual existe un registro detallado de los sentidos posibles del verbo 
en cuestión; además, se elicitarán los datos con nuestra intuición idiomática particular como hablante nativa de castellano. Consideramos importante el contraste directo de datos relativos a las manifestaciones semánticas del verbo de movimiento $i r$, a fin de definir la red relacional posteriormente. Un aspecto relevante es la obtención de enunciados apropiados en los cuales se evidencie la expresión de sentidos en usos manifiestos; es decir, requerimos de datos de la lengua en uso a fin de obtener pistas sobre las posibles relaciones cognitivas. Una cuestión importante es la relacionada con la obtención de datos en el marco de la LC. No existe un consenso sobre las fuentes, puesto que ciertos investigadores consideran que se pueden abstraer a partir del conocimiento del funcionamiento lingüístico, mientras que otros se inclinan por la recopilación de evidencia real (la lengua en uso en contextos de interacción reales). Nuestro propósito es más amplio, pues realizaremos un trabajo de recolección con la consiguiente confrontación con nuestras intuiciones propias como hablantes de castellano; en otras palabras, a fin de realizar esta fase de recolección de evidencia lingüística en la que se presenten sentidos no convencionales, nos permitimos la confrontación con nuestra conocimiento de la lengua castellana y el comportamiento dialectal particular.

El establecimiento de la red de significados del verbo de movimiento ir se realizará mediante la determinación de cuáles de estos se aproximan al significado prototípico de movimiento en términos espaciales. Es decir, se analizarán de acuerdo con cuáles mantienen, ya sea mediante mecanismos metafóricos o metonímicos, el sentido físico vinculado con la dimensión espacial. La finalidad de este procedimiento es establecer la red relacional mediante los diversos usos, lo que equivaldría a diferentes significados que hacen posible, como se explicará en el capítulo siguiente, la experimentación y la organización de la realidad. En resumen, el procedimiento metodológico fundamental constará de dos fases medulares (aunque no sean las únicas):

a) Elaboración del estado del arte sobre el verbo ir en castellano. A fin de iniciar el trabajo de investigación, se realizó un rastreo respecto de los trabajos previos que se han realizado sobre el verbo de movimiento ir.

b) Recopilación de datos. Los distintos usos del verbo ir, manifestados en expresiones concretas, se recopilarán principalmente del repositorio de verbos Adesse de la Universidad de Vigo, que consiste en un 
banco de registro de diferentes significados de tipo verbal. Este banco virtual relativo a verbos del castellano de la Universidad de Vigo:

es una base de datos de verbos y construcciones verbales del español con el análisis sintáctico-semántico de un corpus, y que permite ofrecer para cada verbo una completa caracterización sintáctico-semántica, con sus alternancias de diátesis junto con las frecuencias relativas de cada alternativa construccional para relaciones semánticas similares.

Adesse, una versión ampliada de la Base de Datos Sintácticos del español actual (BDS) que contiene información sintáctico-semántica sobre las cláusulas y los verbos registrados en un corpus del español (Arthus) de 1,5 millones de palabras 4 .

Este repositorio de formas verbales será fundamental, en principio, para reconocer los diversos usos del verbo de movimiento ir, además de ser una buena herramienta inicial para realizar el análisis y las relaciones semánticas consistentes con el verbo en cuestión.

c) Análisis de datos. El análisis de la evidencia de usos verbales relativos al verbo de movimiento ir se realizará en el marco de los presupuestos desarrollados por la LC respecto de la polisemia.

\section{Marco teórico}

\subsection{La LC y el lenguaje como mecanismo generador de significados}

La LC como abordaje teórico deslinda de la tradición generativa fundada por Noam Chomsky en la segunda mitad del siglo XX. El giro de este enfoque se evidencia en la consideración de que el lenguaje no es innato. Las implicancias de esta asunción suponen la consideración de que el lenguaje, en tanto capacidad, forma un continuo con las distintas habilidades cognitivas humanas (Moreno Mojica, 2015). En términos generales, la autonomía lingüística implicada en la modularidad de naturaleza sintáctica es desplazada por la consideración de que el lenguaje sirve para definir y explicitar significados, de manera que se evidencia cierta afinidad con las propuestas funcionalistas (Goldberg, 1996, pp. 3-4). Dicho esto, el núcleo duro del generativismo (esto es, la tesis innatista) enfrenta una fuerte oposición. En rigor, autores como Langacker (2008), en su obra de introducción de la propuesta cognitiva del lenguaje, desarrolla parte del marco 
teórico-conceptual que asumiremos en el presente trabajo. En términos generales, la lingüística cognitiva sostiene la dependencia de la facultad del lenguaje respecto de la cognición general, de manera que la autonomía asumida por los modelos generativos formalistas no se sostiene. Esto implica que las habilidades cognitivas como la percepción, la emoción, el razonamiento, la memoria, entre otras, interactúan con el lenguaje. De manera concreta, la propuesta cognitiva propone que la interacción entre la percepción y el concepto (conception) presenta fuertes implicancias. Esta integración del lenguaje, concebido como una habilidad cognitiva humana distintiva, con otras habilidades cognitivas es lo que distingue la LC de la lingüística cognitiva (con "c" y "l” minúsculas). Esta última, en palabras de Taylor (2003, p. 5), está referida "a cualquier teoría lingüística que reconoce el hecho de que el lenguaje reside en la mente, independientemente de si la teoría acepta la premisa de que el conocimiento lingüístico debería integrarse con todo cuanto se conoce ya acerca de la cognición humana”. A pesar de la fuerte oposición entre el cognitivismo y el generativismo, es preciso indicar que la impugnación de la autonomía lingüística no se equipara a la negación de la existencia de una capacidad innata para el lenguaje; sobre el particular, W. Croft y A. Cruse $(2004$, p. 2) afirman sería erróneo considerar que tal impugnación es una "negación de una capacidad innata para el lenguaje", dado que "únicamente [se asume] como la negación de una capacidad humana innata para el lenguaje con carácter autónomo y con funcionalidad para cualquier uso especial”. En la misma línea se manifiesta Goldberg, quien defiende que "el conocimiento de la lengua es conocimiento" (1995, p. 3). En este sentido, debe subrayarse que la LC no está principalmente interesada en el descubrimiento de un conjunto de universales lingüísticos, sino más bien en entender cómo la cognición humana motiva determinados fenómenos lingüísticos, que se describen como tendencias o generalizaciones abstractas más que como reglas absolutas. Los autores cognitivistas esgrimen argumentos adicionales en contra de la autonomía del sistema lingüístico recurriendo a la ausencia de una separación absoluta entre el conocimiento lingüístico y el conocimiento enciclopédico, que a su vez revela la coherencia existente entre los procesos de cognición lingüísticos y no lingüísticos. Así pues, el significado lingüístico, que implica conocimiento del significado y de la forma, se considera parte del sistema conceptual general del hablante de una lengua y no un módulo o nivel separado.

La lingüística cognitiva comprende dimensiones de la experiencia humana, construidas a través del lenguaje, a partir de la experiencia individual, colectiva, 
social y cultural, de modo que abarca las diferencias entre culturas, grupos sociales o incluso individuos. Esta experiencia humana tiene sus propios aspectos colectivos e interactivos, por lo que reconoce la esencia sociointeractiva del lenguaje, así como su enfoque social como características integrales del cognitivismo. Vale la pena recordar que, desde esta perspectiva, es a partir de estas experiencias humanas experimentadas por cada individuo que dichos hablantes conceptualizan o categorizan ciertas estructuras lingüísticas. Desde el momento en que la cognición comienza a tener en cuenta los aspectos sociales, inevitablemente tiene en cuenta la variación inherente a los idiomas, ya que la variación es la consecuencia inmediata e inevitable del uso. Nunca una comunidad lingüística es totalmente homogénea, por lo que no hay forma de evitar la variación lingüística desde el momento en que un modelo basado en el uso se toma en serio y en su totalidad.

Dentro de este ámbito de estudios cognitivos, es esencial resaltar el proceso de categorización, que es una de las capacidades fundamentales que abarca el proceso mental de identificación, clasificación y denominación de diferentes entidades, como miembros de la misma categoría, que se basa en prototipos. La categorización es una de las capacidades humanas que influye en la actividad sociocontextual, además de crear expectativas en nuestra relación con el medioambiente y constituyen formas de percepción. De acuerdo con los estudios realizados dentro del alcance de la categorización, tenemos que nuestras categorías están condicionadas por la herencia cultural que poseemos por los esquemas culturales que heredamos. En este marco de estudios cognitivos, es esencial resaltar el proceso de categorización, que es una de las capacidades fundamentales que abarca el proceso mental de identificar, clasificar y nombrar diferentes entidades como miembros de la misma categoría, que se basa en prototipos. La categorización es una de las capacidades humanas que influyen en la actividad sociocontextual, así como la creación de expectativas en nuestra relación con el medioambiente y la formación de formas de percepción. De acuerdo con los estudios realizados dentro del alcance de la categorización, tenemos que nuestras categorías están condicionadas por la herencia cultural que poseemos por los esquemas culturales que heredamos.

Los presupuestos teóricos sostenidos por la LC se centra en el significado; en consecuencia, dista del carácter sintactocéntrico de la tradición generativa: "La función básica del lenguaje es expresar significado. Una gramática debería por tanto mostrar de la forma más directa posible cómo los parámetros de forma se unen con parámetros de significado" (Lakoff, 1987, p. 583). Aun cuando el 
significado es el objetivo primordial de estudio, los aspectos formales de la lengua son asumidos de forma consistente, de manera que no debe interpretarse como una simple permutación de niveles, o la predominancia de un nivel en desmedro de otros. La LC se interesa en el uso, aunque metodológicamente los datos o el corpus no necesariamente se constituyen a través de usos lingüísticos reales. Así, la fuerza lingüística semántica que dinamiza la evidencia tanto sintáctica como morfológica es la semántica de las lenguas; en tal sentido, la descripción de estos niveles debe estar signada por la explicitación de las estructuras simbólicas que las direccionan. Es por ello que el enfoque asumido en la presente tesis será el desarrollado en el seno de la LC, a fin de definir la estructura simbólica de los verbos de movimiento. Además, las representaciones semántico-pragmáticas se vinculan directamente con los aspectos formales, sin necesidad de invocar niveles de sintaxis ocultos, transformaciones o derivaciones. La LC defiende que la semántica no es autónoma de la pragmática, el contexto y la cognición. De hecho, la LC concibe la relación entre forma y significado como una correspondencia motivada y no arbitraria, entendiendo que la motivación proviene de una serie de factores, que a menudo entran en competición, y entre los cuales juegan un papel primordial los principios icónicos de organización lingüística. Además, como observa Fauconnier (2004, p. 661), una importante consecuencia de desdibujar la distinción entre semántica y pragmática es que "las teorías cognitivas ponen de relieve la centralidad de las operaciones de tipo pragmático en la construcción del significado". En consonancia con ello, la LC tiene en consideración aspectos detallados del pensamiento humano, los procesos cognitivos y la comunicación social, ya que se entiende que "los cerebros y las culturas desempeñan un papel fundamental a la hora de construir el mundo tal y como lo percibimos" (Fauconnier, 2004, p. 567). Dado que la forma lingüística se considera que viene determinada en alto grado por los procesos de cognición y de comunicación, la LC niega asimismo la autonomía de la sintaxis con respecto a la semántica o la pragmática.

Además, se asume que el significado está corporeizado (embodied), es decir, arraigado en nuestra experiencia corpórea, tal y cómo esta se procesa a través de la percepción y la cognición y que se elabora posteriormente por medio de la metáfora, la metonimia y la integración conceptual (blends). El significado está presente en todos y cada uno de los elementos que conforman el inventario de unidades y fenómenos lingüísticos, por lo que no puede decirse que exista elemento alguno que no aporte una carga semántica. De igual forma, la LC defiende una visión del significado muy dinámica y muy creativa, que, en palabras de Fauconnier (2004, 
p. 661), puede definirse como "un número básicamente ilimitado de formas en que una expresión puede desencadenar procesos cognitivos dinámicos, que incluyen conexiones conceptuales, correspondencias, combinaciones y simulaciones" (Fauconnier, 2004, p. 661). Además, la capacidad para concebir la realidad lingüística de formas alternativas (construal), la categorización por prototipos y extensiones de los prototipos, así como la polisemia, se considera que desempeñan un papel primordial en la conceptualización y, por ende, en el significado. Este último concepto, el de polisemia, será fundamental para efectos del análisis posterior que realizaremos sobre los sentidos del verbo de movimiento ir. Por tal razón, será desarrollado en el subcapítulo § (7.3).

\subsection{El lenguaje en el espacio basado en el uso}

Los presupuestos de la sociolingüística cognitiva serán asumidos en la presente investigación. Se parte de la premisa de que el "lenguaje es una capacidad humana que se manifiesta en forma de variedades lingüísticas, que se utiliza para la comunicación con fines diversos, que se ejercita de manera colectiva y cuyo origen y configuración están íntimamente relacionados con la vida social" (Moreno Fernández, 2012, p. 23). Eso supone que el lenguaje, más que una facultad especializada que opera al margen de la cognición, está íntimamente integrada con esta. En cuanto al funcionamiento lingüístico, algunos de los principios que se proponen en la propuesta cognitiva, se definen los siguientes (Moreno Fernández, 2012, p. 24):

a) Los usos lingüísticos son realidades emergentes, producidas y percibidas como tales.

b) Los usos lingüísticos son esencialmente variables y reflejan la forma de las lenguas a la vez que la determinan.

c) Los usos lingüísticos se producen en escenarios discursivos, entendidos como modelos cognitivos de interacción verbal que surgen en contextos específicos de una realidad social y que están integrados por secuencias de actos de habla.

d) La lengua es un sistema adaptativo complejo de uso dinámico, en el que los procesos de adquisición, de uso y de cambio lingüísticos no son independientes entre sí, sino aspectos de un mismo sistema. 
e) La cooperación comunicativa interindividual convierte la lengua en un fenómeno emergente, con una existencia social crecientemente compleja y una existencia individual basada en factores de naturaleza cognitiva, psicomotriz, perceptiva y experiencial, de modo que las variantes preferidas por el uso lingüístico social acaban integrándose gradualmente en la mente individual.

La importancia de este modelo basado en el uso es que, en la interacción comunicativa como escenario en el cual se desarrollan verbalizaciones del conocimiento lingüístico en relación con otros marcos de conocimiento motivados por la experiencia, emergen de manera dinámica los significados en términos sociales y culturales. Además de estas afirmaciones principales de la sociolingüística, entendida en el marco de la propuesta cognitiva sobre el lenguaje humano, se entiende que las lenguas se dinamizan a través de los hablantes quienes están completamente influidos por la presión generada de los componentes culturales, la comunidad, el grupo al cual se pertenece, etc. Por otro lado, son los contextos socioculturales erigidos por los individuos a través de la interacción verbal los que se constituyen en mosaicos de interpretaciones divergentes en los cuales los individuos se sitúan y cohabitan. Así, la red de relaciones entre los hablantes y sus respectivas comunidades dependen en gran medida del componente dimensional y temporal de los entornos comunicativos que se generan a través del lenguaje, de manera que se gestan valoraciones diversas de los contextos socioculturales, pues los que se perciben como más amplios se valoran de forma más positiva. Uno de los tantos entornos es la ciudad, la cual es valorada debido a que en esta se gestan intercambios lingüísticos (los cuales incluyen o involucran elementos tanto sociales como culturales).

Así, la experiencia de los hablantes es fundamental, pues gracias a esta se constituyen esquemas a través de los cuales los hablantes de una lengua categorizan la realidad. Los conocimientos previos que suponen la constitución de esquemas permiten que las nuevas experiencias vayan ajustándose a estos modelos cognitivos y, en tal sentido, el uso concreto en acciones discursivas son los que definen las variaciones de sentido o significado en una comunidad lingüística. Por ello, es posible que podamos asumir la red de significados del verbo de movimiento ir de acuerdo con estos presupuestos: "En tal sentido, es pertinente delimitar ciertos principios (o criterios) que definen el papel de la experiencia en la instalación de esquemas conceptuales que, finalmente, definirán la ocurrencia de esquemas 
metafórico-conceptuales, los cuales, a su vez, derivan en la presencia de unidades fraseológicas en situaciones concretas de habla”(Moreno Fernández, 2012, p. 45).

Habiendo delimitado los aspectos principales de la propuesta cognitiva del lenguaje, pasaremos a desarrollar de manera puntual los aspectos vinculados con la polisemia.

\subsection{La polisemia como redes de significado}

El análisis de la polisemia ha devenido en el marco de la LC en la consideración principal de que el significado está motivado por la experiencia de los hablantes. Una primera mirada sostiene que el significado se manifiesta a través de sentidos que se reconocen en contextos específicos. Esta conjetura le confiere un valor semántico al uso que se expresa en el contexto comunicativo o la experiencia concreta; sin embargo, no es claro cuál sería el estatus del significado, pues este solo es analizado en la concreción. Por ello, consideramos que, si bien la LC asume el significado asociado con la cognición general y, en tal sentido, el mecanismo de conceptualización y la experimentación del mundo físico, permiten el acceso a otros marcos de conocimiento que involucran una mayor complejidad conceptual. Por ello, nos permitimos asumir la asunción de la teoría de prototipos, la red semántica, entre otros, para explicar la polisemia del verbo ir en castellano.

\section{Análisis de datos}

Los datos relativos a los significados del verbo de movimiento ir serán recogidos de Adesse. La relación de sentidos posibles se presentará de acuerdo con cada uno de los significados que plantea el repositorio. Posteriormente, consideraremos la conformación de una red en la que se represente el significado nuclear del cual se dispersan otros que no necesariamente se vinculan con este. Para que la evidencia siga un orden propondremos algunos enunciados a fin de razonarlos en el marco de un estudio que ofrezca una visión integrada de las diferencias semánticas. Iniciaremos la exposición con el sentido paradigmático del verbo ir; es decir, el significado relativo a la dimensión de tipo físico-espacial. Este se presentará a través de algunas expresiones derivadas del rastreo realizado en Adesse.

(2) a. Fui al concierto el fin de semana.

b. Aún no se vayan de la fiesta, por favor. 
c. E1 trayecto del bus $\boldsymbol{v} \boldsymbol{a}$ de Lima a Santa Beatriz.

d. Siempre se nos va el tiempo demasiado rápido.

e. El tiempo de espera $\boldsymbol{v a}$ de seis a siete semanas.

En los casos anteriores, existe una relación espacial en los tres primeros casos, en el sentido de que se trata de trayectoria. Así, en la expresión (2a) se alude a desplazarse hacia un lugar en particular. En (2b) la dirección cambia, pues se trata de desplazarse desde un punto hacia un lugar indeterminado, aunque en este caso se mantiene el sentido relativo al espacio. El sentido de (2c) se refiere a una trayectoria con dos puntos fijos. Finalmente, los casos presentados en (2d) y (2e) también mantienen el sentido de desplazamiento o trayectoria, pero en términos metafóricos; es decir, se establece la metáfora El tiempo es trayectoria. De esta manera, se integran los cinco casos en los cuales la dirección espacial puede incluir al tiempo. A continuación, se analizarán las expresiones vinculadas con el sentido II del desenvolvimiento de algo. Veamos los datos de (3).

(3) a. Iremos con la actitud de siempre a la reunión.

b. Me $\boldsymbol{v} \boldsymbol{a}$ bien con el italiano en ese instituto.

c. Contra todo pronóstico, nos fue bien.

Los casos anteriores implican dinamicidad en el sentido de desarrollo de un evento concreto. Así, el desarrollo se interpreta espacialmente también de acuerdo nuevamente con un mecanismo metafórico. Por ello, el primer dato está vinculado con el comportamiento: se trata de la forma en que se comporta o se encuentra alguien en términos conductuales. En cuanto a la expresión de (3b), esta supone el desenvolvimiento de una determinada manera o el funcionamiento de algo (nuevamente el ámbito del desarrollo de un evento en este caso); finalmente, en (3b) el significado se enfoca en el resultado, ya sea este adecuado o inadecuado. A pesar de que es claro que los tres ejemplos presentados carecen de relación alguna con la realidad física, la relación que proponemos implica la activación de una metáfora conceptual en el cual El desarrollo de Eventos es Movimiento. En atención a lo dicho, ya sea que se trate del comportamiento, del funcionamiento o del desenvolvimiento, en todos los casos existe un sentido direccional cuya motivación proviene del mundo físico. En cuanto al tercer sentido, este presenta una clara función relacional y su sentido es el de armonizar o coincidir, es decir, está referido a la preferencia, la inclinación por algo o por alguien. A continuación, se incluyen los siguientes datos en (4): 

a. A mí no $\boldsymbol{m e} \boldsymbol{v} \boldsymbol{a}$ tu manera de pensar.
b. Le voy al Alianza Lima este fin de semana.
c. Le voy a la vida despreocupada.

Cada uno de los casos se asocia con las preferencias personales, las tendencias, las apreciaciones de carácter subjetivo. En cuanto a las expresiones, estas suponen direccionalidad en el sentido de que se expresa una orientación hacia un punto en concreto que es inmaterial, pero que se conceptualiza como material. Las preferencias entonces se interpretan a mediante el dominio del movimiento; en consecuencia, el verbo ir es importante para establecer las asociaciones semánticas consistentes con esta variación del verbo. Sustancialmente, planteamos la metáfora, Las preferencias son movimientos. Por ello, es posible incluir estos últimos en la red de significados del verbo $i r$. Un caso adicional agrupa expresiones referidas a la dimensión sensorial. Veamos los siguientes casos:
a. Creo que le $i b a$ bañarse con agua fría.
b. Me va Jorge porque tiene buen corazón.
c. No te van las películas de terror.

Los casos presentados obedecen al sentido o significado de gustar. Aunque podría parecer que carece por completo de relación. El gusto define un objetivo (aquello que genera o desencadena el gusto); por ello, asumimos que cada uno de los casos se ajusta al sentido de direccionalidad o desplazamiento, aunque en estos casos el objetivo del desplazamiento sea heterogéneo (puede ser un evento como bañarse con agua fría, una persona como Jorge, etc.). Las tres expresiones estarían definidas por la metáfora LAs SENSACiONES SON MOVimientos. En otros términos, es la direccionalidad física la que permite interpretar los fenómenos sensoriales de acuerdo con la característica metafórica de expresar lo complejo a través de lo simple. Finalmente, existe un caso adicional vinculado con el mundo fisiológico. Este se propondrá a continuación en (6):

(6) a. Después de comer apurado el cebiche, se vino en diarrea.

b. Todos nos vinimos en diarrea. 
Las expresiones del sentido $\mathrm{V}$ del verbo de movimiento ir acude a un mecanismo metonímico: el individuo por las excretas. Un caso adicional de la forma en que la direccionalidad espacial permite configurar el padecimiento de afecciones gastrointestinales.

\subsection{Teoría de prototipos}

En cuanto a la teoría de prototipos, se considera que existe un elemento nuclear del cual irradian los significados, desde los que más se aproximan al centro hasta los que se alejan del significado central. Sostenemos que se forman esquemas de imágenes que definen direccionalidad espacial de diverso tipo para referirse tanto a eventos físicos como no físicos y, para ello, se usa el verbo ir. A continuación se proponen algunos casos relativos a los ejemplos anteriores.

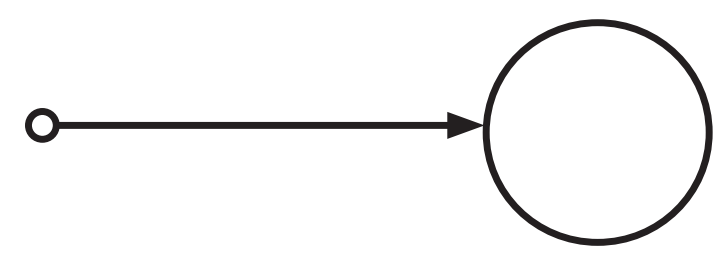

Figura 1. Esquema de imagen de IR A. Elaboración propia.

Sin embargo, también es posible usar el verbo ir para definir una dirección indeterminada. Es decir, desde un espacio concreto hacia fuera. A continuación, proponemos el esquema de imagen.

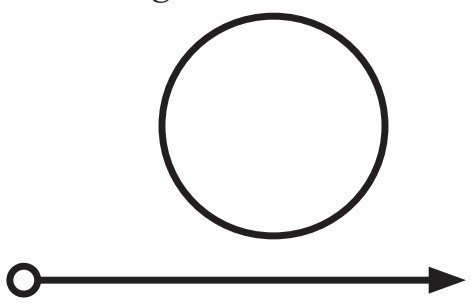

Figura 2. Esquema de imagen de IR DE. Elaboración propia.

No son los únicos esquemas en cuestión; sin embargo, es a partir de estas coordenadas espaciales que se configuran los significados a través de una red polisémica, la cual planteamos a continuación. Usaremos el modelo básico de prototipos (Valenzuela, Ibarretxe-Antuñano y Hilferty, 2012, p. 57). 


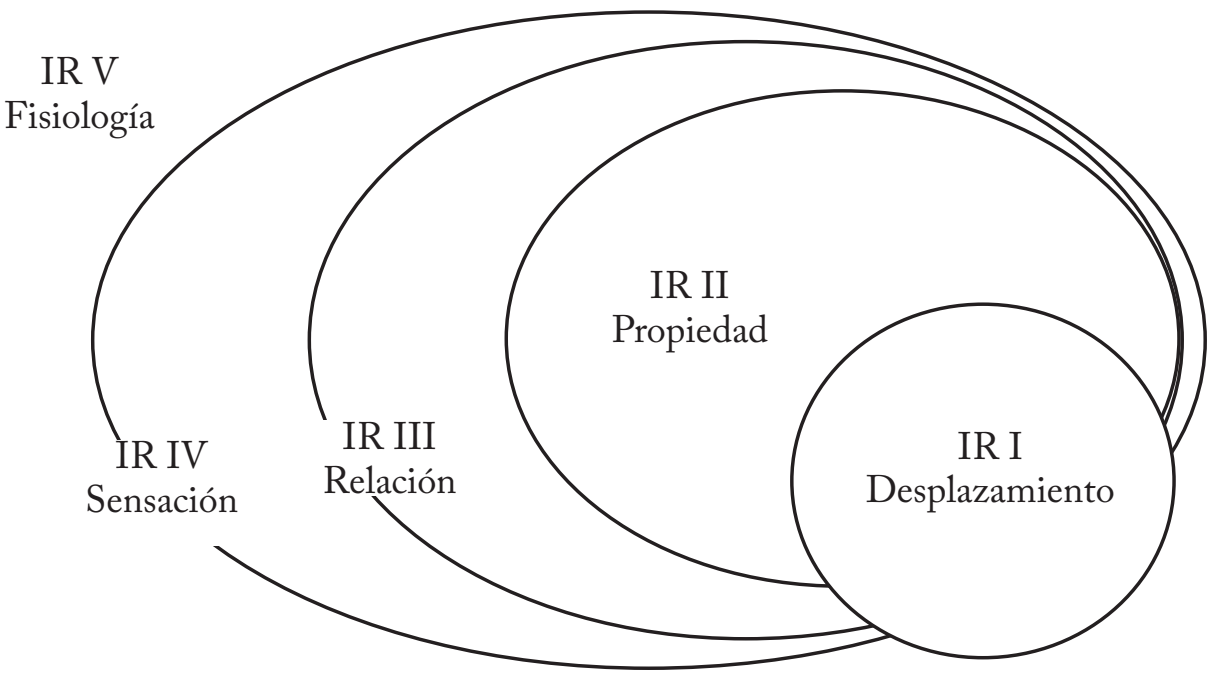

Figura 3. Relación prototípica del verbo de movimiento IR. Elaboración propia.

El caso anterior permite reconocer la relación entre los significados de manera integrada. En tal sentido, consideramos que la polisemia de ir es explicable en términos de mecanismos metafóricos motivados por la experiencia espacial. En tal sentido, es posible suponer que la conformación de esquemas motivados por la percepción física genera mecanismos metafóricos para definir diversos tipos de expresiones en los cuales la dimensión física se extiende hacia dominios diferentes (sensaciones, emociones, tiempo, etc.).

En conclusión, consideramos pertinente la relación semántica entre significados, con lo cual se sostiene que la cognición asegura la estructura de significados, aun cuando parecieran algunos carecer de relación, estos son sensibles a la correlación; sin embargo, el estatus teórico de tales relaciones debe ser replanteado a través de los prototipos que se van gestando, y que son el mecanismos idóneo para que la plasticidad y dinamicidad del significado se exprese.

\section{Conclusiones}

1. El verbo ir en castellano se organiza a través de múltiples significados dispuestos en una red semántica, en la cual, a partir de un significado central o punto de referencia cognitivo, se estructuran otros significados más o menos próximos. 
2. E1 significado prototípico del verbo ir en castellano explicita la experimentación espacial; es decir, se asocia con un referente físico. A partir de este sentido central es que irradian otros vinculados con eventos, preferencias, entre otros.

3. La red semántica del verbo ir se configura a través de mecanismos metafóricos que direccionan la comprensión de dominios de mayor complejidad como los procesos fisiológicos o las preferencias. De esta forma, la experiencia física es el canal mediante el cual se conceptualizan otras experiencias a fin de simplificar su expresión a través de la lengua.

4. El camino de la expresión de lo complejo a través de lo simple es una muestra del principio de economía cognitiva; esto es, mediante un significado sencillo que supone direccionalidad, se hace posible la interpretación plena de procesos o de eventos de mayor complejidad.

\section{Notas}

1 El presente artículo es producto de un trabajo de tesis más amplio en el cual se razonan las metáforas en verbos de movimiento en castellano, como requisito para la obtención del grado de magíster en Lingüística por la Universidad Nacional Mayor de San Marcos.

2 Este repositorio de datos verbales de la Universidad de Vigo se puede consultar en el siguiente enlace: http://adesse.uvigo.es/

3 Los datos relativos al verbo ir han sido extraídos de Adesse: http://adesse.uvigo.es/ data/verbos.php?verbo=ir

4 Recuperado de Adesse: http://adesse.uvigo.es/index.php

\section{Contribución del autor}

Gisela Silva Escudero ha participado en la elaboración, la compilación de datos, la redacción y el consentimiento de la versión final del presente artículo.

\section{Fuente de financiamiento}

Este trabajo es autofinanciado.

\section{Conflictos de interés}

Ninguno. 


\section{Trayectoria académica}

Gisela Silva Escudero es bachiller en Lingüística por la Universidad Nacional Mayor de San Marcos y candidata a magíster por la misma universidad. Sus intereses investigativos giran en torno a la semántica del castellano, lengua en la que ha realizado pesquisas sobre la naturaleza cognitiva de las formas verbales. Además, es correctora de textos y profesora a nivel preuniversitario y universitario.

Referencias bibliográficasCroft, W. y Cruse, A. (2004). Cognitive linguistics (Cambridge Textbooks in Linguistics). Cambridge University Press.

Evans, V. y Melanie G. (2006). Cognitive linguistics. An introduction. Edinburgh University Press.

Evans, V.y Paul C. (eds.) (2010). Language, cognition and space: The state of the art and new directions. Equinox.

Evans, V. y Stéphanie, P. (eds.) (2009). New directions in cognitive linguistics. John Benjamins Publishing Company.

Fauconnier. G. (2004). Mental spaces, language modalities, and conceptual integration. In S. Davis y B. Gillon (eds.). Semantics: A reader. Oxford University Press.

Filipczuk-Rosińsk, S. (2016). The comparison of A HuMAN BEING Is A PLANT metaphor between the English and Polish language. World Journal of Social Science, $3(1), 15-21$.

Goldberg, A. E. (1996). Jackendoff and construction-based grammar. Cognitive Linguistics, 7, pp. 3-19.

Ibarretxe-Antuñano, I. y Valenzuela, J. (2012). Lingüistica cognitiva. Anthropos Editorial.

Ibarretxe-Antuñano, I. (1999). Polysemy and metaphor in perception verbs: A cross-linguistic study. [Tesis de doctora en Lingüística, Universidad de Edimburgo]. https://ethos.bl.uk/OrderDetails.do?uin=uk.bl.ethos. 652784

Jackendoff, R. (2002). Foundations of language. Oxford University Press.

Kövecses, Z. (2010). Metaphor: A practical introduction. Oxford University Press.

Lakoff, G. y Turner, M. (1989). More than cool reason: a field guide to poetic metaphor. University Chicago Press. 
Polisemia y cognición del verbo ir en castellano

Lakoff, G. (1987). Women, fire and dangerous things: What categories reveal about the mind. Chicago University Press.

Lakoff, G. y Johnson, M. (1980). Metaphors we live by. University of Chicago Press.

Langacker, R. (2009). Investigations in cognitive grammar. Mouton de Gruyter.

Langacker, R. (2008). Cognitive grammar: A basic introduction. Oxford University Press.

Moreno F. (2012). Sociolingüistica cognitiva. Proposiciones, escolios y debates. Iberoamericana.

Taylor, J. R. (2003). Cognitive grammar. Oxford University Press. 